Objective: Both early-onset Alzheimer's disease $(E O A D)$ and late-onset Alzheimer's disease (LOAD) present with cognitive and psychiatric features. Some studies suggest that EOAD patients are more likely than LOAD patients to have psychiatric symptoms. If this is true, relatives of EOAD patients with a similar clinical presentation may be more likely to be misclassified as having a primary noncognitive psychiatric disorder rather than a dementing disorder. Family history studies may underestimate familial aggregation of $E O A D$. Methods: The authors compared the presence of psychiatric symptoms in parents and siblings of 131 EOAD patients (diagnosed at or before age 60), with the parents and siblings of 131 LOAD patients (diagnosed at or after age 65). Early onset Alzheimer's disease and LOAD patients were matched for diagnosis (probable versus possible AD), gender, and ethnic group. Logistic regression analysis was performed on the outcome variable of patient group (EOAD, $L O A D$ ) with family history of psychiatric symptoms as the risk factor, adjusting for family size and patient's education. Results: There was a

\section{A Comparison of Family History of Psychiatric Disorders Among Patients With Early- and Late-Onset Alzheimer's Disease}

Gayatri Devi, M.D.

Jennifer Williamson, M.Sc.

Fadi Massoud, M.D.

Karen Anderson, M.D.

Yaakov Stern, Ph.D., D.P.

D. P. Devanand, M.D.

Richard Mayeux, M.D., M.Sc.

nearly two and one-half-fold increase in family history of psychiatric symptoms among EOAD patients when compared with LOAD patients (RR 2.4; 95\% C.I. 1.2-4.7). Conclusions: The authors found preliminary evidence of a higher prevalence of a history of psychiatric symptoms among relatives of EOAD patients when compared to LOAD patients. This may be due to differential misclassification of $A D$, a syndromic disorder with both noncognitive psychiatric and cognitive deficits in relatives of EOAD patients. Alternatively, shared genetic or other familial etiologies may underlie subtypes of EOAD and some psychiatric disorders.

(The Journal of Neuropsychiatry and Clinical Neurosciences 2004; 16:57-62)

Received December 12, 2001; revised July 9, 2002; accepted October 15, 2002. From the New York Memory and Healthy Aging Services (G. Devi), the Departments of Medicine (Neurology) (G. Devi) and Psychiatry (G. Devi) of Lenox Hill Hospital, G. H. Sergievsky Center (J Williamson, F Massoud, K Anderson, Y Stern, R Mayeux); the Department of Neurology (K Anderson, Y Stern, R Mayeux) and Psychiatry (K Anderson, DP Devanand) and the Taub Alzheimer's Disease Research Center (K Anderson, Y Stern, DP Devanand, R Mayeux) of Columbia University. Address correspondence to Dr. Devi, The NY Memory and Healthy Aging Services, 65 East 76th St., New York, NY 10021; gd@nymemory.org (E-mail).

Copyright (C) 2004 American Psychiatric Publishing, Inc. 
$\mathrm{B}_{\mathrm{h}}^{\mathrm{o}}$ oth the genotype and the clinical phenotype of Alzheimer's disease (AD) are heterogeneous, with wide variations in the age of onset, clinical manifestations, and the presence of known genetic mutations, all resulting in the common neuropathologic hallmarks of AD. ${ }^{1-4}$ Especially striking are the disparities in the known genetic etiologies and clinical course between early-onset AD (EOAD) and late-onset AD (LOAD), despite little neuropathological distinction. ${ }^{5}$

Mutations of several genes, most commonly presenilin 1, have been described in EOAD ${ }^{1-2}$ and account for about one-half of all EOAD cases. Late-onset AD, with an age of onset after 65 years, has been most consistently associated with the $\varepsilon-4$ isoform of the apolipoprotein $\mathrm{E}$ gene. ${ }^{6}$ Much remains to be determined regarding the genetics of $\mathrm{AD}$.

Clinically, AD is a multispectrum brain disorder with psychiatric, movement, and cognitive abnormalities. Several studies have noted differences in clinical characteristics between early- and late-onset AD patients, particularly in symptoms and disease course. Even within EOAD kindreds, there are clinical variations in characteristics such as disease duration and the presence of myoclonus. $5,7,8$ Some EOAD patients exhibit prominent psychiatric features, with $41 \%$ of patients experiencing mild EOAD manifesting psychiatric symptoms in one study. ${ }^{8-10}$ Others have found an increased risk for a family history of depression among depressed LOAD patients. ${ }^{11-12}$ Additionally, AD patients with a family history of dementia were more likely to have a family history of a psychiatric disorder than AD patients without a family history of dementia. ${ }^{13-14}$

Because of the presence of both cognitive and psychiatric features in $\mathrm{AD}$, inquiry into the presence of psychiatric symptoms and disorders in families of patients with AD may be helpful in family and genetic studies. Significant differences in the prevalence of psychiatric symptoms between EOAD and LOAD families may imply differential misclassification of a primary dementing disorder as a primary nondementing psychiatric disorder. Alternatively, such a difference may implicate shared genetic influences amongst subtypes of psychiatric symptoms and subtypes of $\mathrm{AD}$. The assessment of familial aggregation of specific clinical disease manifestations may lead to a more precise delineation of subtypes of familial $\mathrm{AD}$, resulting in a more homogeneous phenotype for genetic analysis.

Accurate identification of affected relatives is important in understanding the genetics of $\mathrm{AD}$ and exploring the roles of genetic and environmental risk factors. Family history studies in $\mathrm{AD}$ and other illnesses are prone to underestimation. ${ }^{15-17}$ If informants are not queried about illnesses such as psychiatric disease in the relative, the true familial aggregation of AD may be underestimated. Misclassification of a relative as primarily psychiatric instead of cognitive can occur, given the extensive clinical spectrum of this brain illness. The discovery of familial co-aggregation of AD with another psychiatric illness may offer clues to understanding the multifactorial etiology of AD.

Aim

We wished to examine if patients with EOAD would have a greater risk for a family history of psychiatric symptoms and disorders (among parents and full siblings) compared to patients with LOAD.

\section{MATERIALS AND METHODS}

Patients were drawn from the Columbia University Alzheimer Disease Research Center (ADRC) database. A total of just over 2,100 patients with cognitive complaints were seen at the time of this study. Consent for enrollment into the ADRC was obtained in the manner prescribed by the Institutional Review Board. Initial screening by our clinic coordinator eliminated all patients with primary psychiatric disorders or other neurologic illnesses (e.g., bipolar disorder, Parkinson's disease).

All patients underwent a structured neurological and neuropsychological examination and a battery of laboratory tests. ${ }^{18} \mathrm{~A}$ diagnosis of probable or possible $\mathrm{AD}$, using NINCDS-ADRDA criteria, was made at a consensus conference of neurologists, psychiatrists, and neuropsychologists. ${ }^{19}$ We searched this database for all patients with a diagnosis of probable or possible AD made when the patient was $\leq 60$ years of age. We included patients with very mild $\mathrm{AD}$ (clinical dementia rating scale severity $=0.5^{20,21}$ ).

We then consecutively matched these patients by sex, clinical diagnosis (probable or possible AD), and ethnic group to patients in the ADRC database with a diagnosis of AD made at or after age 65. For both EOAD and LOAD patients, we used age at diagnosis rather than age of onset of disease. Age of onset is a historical feature that is prone to recall bias and varies, depending on the informant as well as the interviewing clinician. Our conservative approach ensured that all those clas- 
sified as EOAD patients had symptoms of illness at or prior to age 60 . We used a 5-year interval to separate the groups in order to minimize overlap in ages of onset between groups.

The evaluating physician performed a clinical family history interview, utilizing both patient and informants at the time of the initial evaluation. In the ADRC, the informant was most often the spouse or child of the patient. ${ }^{17}$ The physician inquired into the presence of psychiatric, memory, and other medical disorders in parents, siblings, and children of patients. The presence of a family history of cardiovascular, neurologic, and other medical conditions was also obtained. The physician noted the diagnosis as conveyed by the informant for entry into the database. If a relative was said to suffer from depression or schizophrenia, for example, they were classified as such. If a relative was reported to have a psychiatric problem, but the informant did not specify a diagnosis, the relative was noted as suffering from an "unspecified mental illness." Similarly, if a relative was noted to have memory problems, but the informant did not report a specific diagnosis, it was entered as "unspecified memory disorder."

A structured family history interview was performed on some patients as part of another concurrently conducted family study. Available autopsy information was obtained.

We analyzed the primary demographic variables using the Student's t test and chi-square methods. We used logistic regression to evaluate the primary outcome variable of EOAD or LOAD, given family history of psychiatric symptoms, memory disorders, and medical conditions, after adjusting for family size and number of years of education of the patient using the SPSS Statistical software package (version 10). We included only family history on parents and siblings as we wished to minimize recall bias with regard to grandparents and other relatives and as the children of both groups were generally not of an age to be at risk. We evaluated the difference in prevalence of other medical and neurological conditions between the groups to examine ascertainment bias on the part of the physician. We adjusted for the patient's education, as this may affect reporting of family history. ${ }^{17}$

\section{RESULTS}

Our database yielded 131 patients with a clinical diagnosis of probable or possible $\mathrm{AD}$ with age at diagnosis of 60 years or less. They were matched by gender, ethnic group, and diagnosis to a comparison group of 131 LOAD patients diagnosed at or after age 65 . Of the 61 women and 70 men in each group, 56\% were Caucasian, 7\% African American, 30\% Hispanic, and the rest were of other or unknown ethnicity.

EOAD patients were about 20 years younger $(55 \pm 5$ years) than their LOAD peers $(76 \pm 6$ years $)(P<0.001)$. EOAD patients ( $13 \pm 5$ years) and LOAD patients $(12 \pm 5$ years) had similar years of education $(p<0.07)$. EOAD patients exhibited a greater tendency toward smaller families $(6 \pm 3)$ than did LOAD families $(7 \pm 3)(p<0.02)$. Family histories were missing in four patients, two from each group. Seven patients had an autopsy (all in the EOAD group), confirming the clinical diagnosis of probable AD in all seven. Eight patients reported a family history of both memory and psychiatric symptoms in the same relative (six in the EOAD group, two in the LOAD group).

Nineteen patients (LOAD, $N=9$; EOAD, $N=10$ ) had an extensive structured family history interview as part of another study, in addition to the physician administered family history. ${ }^{17}$ These structured interviews confirmed the data obtained from the physician's interview in 15 of the 19 cases. Two of the EOAD patients did not report psychiatric illness in the family in the physician's interview, but did so later in the tester-administered structured family history interview. Two of the LOAD patients, who reported psychiatric illness in the family in the physician's interview, did not report this in the structured interview.

In the logistic regression analysis, patients with EOAD had an at least two-fold increase in family history of psychiatric disease compared to patients with LOAD both before ( $R R=2.0 ; 95 \%$ C.I: 1.1-3.6), and after, adjusting for education and family size $(R R=2.4 ; 95 \%$ C.I: 1.2-4.7).

There was no significant difference in family history of memory disorders or illnesses such as other neurological disorders, cancer, or cardiovascular disease between the EOAD and LOAD patients. The presence of psychiatric and other illness in the family is tabulated in Table 1.

\section{DISCUSSION}

In this exploratory study, we found a nearly two and one-half-fold increase in the family history of psychiat- 
ric symptoms among EOAD patients, when compared to LOAD patients after adjusting for possible confounders. This finding may be due to the differential misclassification of $\mathrm{AD}$ as a psychiatric disorder among relatives of EOAD patients. Alternatively, our results could imply that some forms of EOAD and subtypes of psychiatric disorders might share common underlying genetic features.

In our data set of eight patients with reports of memory and psychiatric disorders in the same relative, six patients reported these disorders in relatives of EOAD patients. This lends some credence to the idea that relatives with EOAD with dementia may be more likely to be differentially misclassified as psychiatrically rather than cognitively affected. On the other hand, some studies have reported high frequencies of psychiatric disorders in early-onset AD patients, and thus relatives of EOAD patients with dementia may also be more likely to manifest prominent psychiatric symptoms.

Both early- and late-onset AD are associated with familial aggregation with psychiatric disease. In an early study of 22 patients with LOAD and 24 nondemented controls, Martins et al. ${ }^{22}$ reported a greater occurrence of psychiatric illness in the families of LOAD patients. In another study on primarily LOAD patients (average age of onset 70-71 $\pm 80-9$ years), 172 patients with a family history of dementia were more likely to report psychiatric disorder in the family than in 290 patients without a family history of dementia. ${ }^{13}$ This suggests that the more heritable forms of AD are associated with psychiatric disease and one would predict that EOAD patients might be more likely to have a family history of psychiatric disorder.

Other studies have demonstrated familial co-aggregation of depressive disorders among patients with LOAD and depressive symptoms, again bespeaking an association between subtypes of LOAD and depressive disorders. ${ }^{11,12}$ A recent study of 371 early- and late-onset AD patients (age of onset 46-90 years) found that psychotic symptoms in AD patients showed familial aggregation amongst siblings with an odds ratio of 2.4. While this study did not evaluate differences in co-aggregation of psychotic symptoms between early- and late-onset $\mathrm{AD}$ patients, the data speak to a clustering of these symptoms amongst families. ${ }^{23}$

Genetic studies have not consistently demonstrated possible associations between subtypes of psychiatric disorders and AD. Genetic linkage studies of psychiatric disorders such as schizophrenia and bipolar disorder have pointed to potential areas of genetic chromosomal overlap with some AD genetic studies, although the results are inconsistent and conflicting. ${ }^{24,25}$ Searches at the amyloid precursor protein locus (APP) in small sets of schizophrenic patients have had mixed results, ${ }^{26}$ and no searches have, as of yet, been performed at the presenilin 1 or 2 locus for primary psychiatric disorders. Genetic studies of these complex psychiatric illnesses and of $\mathrm{AD}$ have proven difficult, primarily due to genetic and phenotypic heterogeneity.

Psychiatric illnesses segregate with other neurogenetic, neurodegenerative diseases. Huntington's disease is a classic neuropsychiatric, neurodegenerative disease, presenting with prominent psychiatric, cognitive, and motor symptoms. ${ }^{27,28}$ Evaluating one type of clinical presentation alone may lead to erroneous conclusions regarding hereditability of this disease. Conversely, a family history of schizophrenia-like symptoms has been shown to segregate with the HD gene. ${ }^{29}$ In the hereditary spino-cerebellar ataxias, ${ }^{30}$ clustering of varying clinical symptoms amongst family members has been associated with distinct mutations. In this latter example, consideration of the familial heterogeneity in clini-

TABLE 1. Family history of memory, psychiatric and other medical disorders (LOAD group was the comparison group).

\begin{tabular}{|c|c|c|c|c|}
\hline & EOAD; $N=129$ & LOAD; $N=129$ & Odds ratio & Odds ratio* \\
\hline Any psychiatric disorder & $34(26 \%)$ & $20(15 \%)$ & $2.0(1.1-3.6)$ & $2.4(1.2-4.7)$ \\
\hline Schizophrenia & $6(5 \%)$ & $2(1.5 \%)$ & $3.1(0.6-15.5)$ & $4.9(0.9-28.3)$ \\
\hline Bipolar disorder & $2(1.5 \%)$ & 0 & Unable & Unable \\
\hline Depression & $4(3 \%)$ & $5(4 \%)$ & $1.0(0.2-3.4)$ & $1.0(0.2-4.9)$ \\
\hline Completed suicide & $5(4 \%)$ & $1(1 \%)$ & $5.2(0.6-44.8)$ & $2.8(0.3-27.8)$ \\
\hline Alcoholism & $15(12 \%)$ & $7(5 \%)$ & $2.3(0.9-5.8)$ & $2.1(0.8-5.4)$ \\
\hline Memory disorders & $43(34 \%)$ & $53(41 \%)$ & $0.7(0.4-1.2)$ & $0.7(0.4-1.2)$ \\
\hline Cardio-vascular disease & $54(42 \%)$ & $55(43 \%)$ & $1.0(0.6-1.7)$ & $0.9(0.5-1.6)$ \\
\hline Malignancies & $40(31 \%)$ & $48(37 \%)$ & $0.8(0.5-1.3)$ & $0.7(0.4-1.3)$ \\
\hline Other neurologic disorders & $6(5 \%)$ & $10(8 \%)$ & $0.6(0.2-1.6)$ & $0.5(0.2-1.5)$ \\
\hline
\end{tabular}


cal phenotype leads to accurate definition of the genotype. These examples illustrate the value of assessing familial phenotype in studying the genetics of complex diseases.

In our study, there was no difference in the prevalence of a family history of memory disorders between EOAD and LOAD patients. A clinic-based study involving 967 first-degree relatives of 128 patients, ${ }^{31}$ found that relatives of patients with EOAD ( $\leq 67$ years) and LOAD ( $>67$ years) had equivalent risks of developing dementia, similar to other such studies. ${ }^{32,33}$

There are several concerns in interpreting our data:

1. Was the diagnosis of $\mathrm{AD}$ accurate in the EOAD group? Could these patients have been misdiagnosed and instead suffer from a primary psychiatric or other dementing disorder (e.g. Huntington's) that is responsible for the seeming increased familial co-aggregation of psychiatric illness? All patients were diagnosed using standardized NINCDS-ADRDA criteria and all 7 autopsied EOAD patients had pathological verification of AD. The sensitivity and specificity of the dementia evaluation compared to pathological diagnosis in our group is $96 \%$ and $85 \%$ respectively. ${ }^{34}$ Thus misdiagnosis seems unlikely to account for our results. Furthermore, patients with primary psychiatric or other chronic neurologic diagnoses were screened out before ADRC evaluation.

2. Was there ascertainment bias: Could EOAD patients have been questioned more thoroughly than LOAD patients? While this is a significant concern, there was no increase in the prevalence of memory disorders between the two groups. In all probability, ascertainment bias would be more strongly directed toward a family history of memory disorders and dementia than towards a family history of psychiatric disorders. Finally, family history rates for all other medical and neurological illnesses were not significantly different between groups, making ascertainment bias unlikely as a significant factor in the results.

3. The prevalence of psychiatric disorders such as schizophrenia varies depending on the decade of the patient's birth (the so-called cohort effect). Did this have a confounding effect on family history rates of psychiatric disorders in EOAD and LOAD patients? In addition, as informants may also be part of the cohort effect, did this have a further impact? This is a limitation of our study.

4. Family members of EOAD patients may be under greater stress and perhaps prone to more adjustment disorder, alcoholism, and depression, and this may explain the higher incidence of psychiatric symptoms in the relatives of EOAD patients. While the interplay between the environment and genetics is well known, this may be another possible confounder of our results.

5. Informants for EOAD patients may have been more likely to have been parents and siblings, whereas informants for LOAD patients may have been more likely to have been children, who may be less able to recall family history in parents and siblings of the patient.

6. Only a subset of our patients was administered a structured family history questionnaire, and the majority received a clinical family history interview. Additionally, no attempt was made to make a formal psychiatric diagnosis. This may have had an impact on the results, although such effects would be similar across both groups.

Our preliminary findings need to be confirmed with a structured family history and psychiatric diagnostic interview, incorporating both memory and psychiatric symptoms in an unselected sample of EOAD and LOAD patients, preferably population based. Confirmation of our findings of familial aggregation of subtypes of EOAD with subtypes of psychiatric disorders may be useful in understanding phenotypic variability of EOAD and may define more homogenous clinical groups for genetic analysis. Alternatively, follow-up of relatives of EOAD patients, looking specifically for cognitive disturbances in the presence of psychiatric disturbances, may be of merit.

Supported by National Institute of Health grant AG08702 and the Taub Research Foundation.

\section{REFERENCES}

1. Van Broeckhoven, CL: Molecular genetics of Alzheimer's disease: identification of genes and gene mutations. Eur Neurol 1995; 35:819.

2. Cruts M, Van Broeckhoven C: Molecular genetics of Alzheimer's disease. Ann Med 1998; 30(6):560-566
3. Cruts M, van Duijn CM, Backhovens $H$, et al. Estimation of the genetic contribution of presenilin-1 and -2 mutations in a populatioin study of presenile Alzheimer disease. Human Molecular Genetics 1998; 7: 43-51

4. Mirra SS: Neuropathological assessment of Alzheimer's disease: 
the experience of the Consortium to Establish a Registry for Alzheimer's Disease. International Psychogeriatrics. 9 Suppl 1:2638; discussion 269-272, 1997

5. Campion D, Brice A, Hannequin D, et al: A large pedigree with early-onset Alzheimer's disease: clinical, neuropathologic, and genetic characterization. Neurology 1995; 45(1):80-85

6. Mayeux R, Saunders AM, Shea S, et al: Utility of the apolipoprotein E genotype in the diagnosis of Alzheimer's disease. Alzheimer's Disease Centers Consortium on Apolipoprotein E and Alzheimer's Disease N Engl J Med 1998; 338(8):506-511

7. Bird TD, Sumi SM, Nemens EJ, et al: Phenotypic heterogeneity in familial Alzheimer's disease: a study of 24 kindreds. urnal Article Annals of Neurology 1989; 25(1):12-25

8. Mullan M. Bennett C. Figueredo C. et al: Clinical features of early onset, familial Alzheimer's disease linked to chromosome 14. Am J Med Genet 1995; 60(1):44-52

9. Mangone CA, Castano EM, Levy E, et al. Early onset Alzheimer's disease in a South American pedigree from Argentina. urnal Article Acta Neurologica Scandinavica 1995; 91(1):6-13

10. Rubin EH, Kinscherf DA, Morris JC: Psychopathology in younger versus older persons with very mild and mild dementia of the Alzheimer type. Am J Psychiatry 1993; 150:639-642

11. Pearlson GD, Ross CA, Lohr WD et al: Association between family history of affective disorder and depressive syndrome in Alzheimer's disease. Am J Psychiatry 1990; 147(4):452-456

12. Strauss ME and Ogrocki PK: Confirmation of an association between family history of affective disorder and the depressive syndrome in Alzheimer's disease. Am J Psychiatry 1996; 153:1340-1342

13. Luchins DJ, Cohen D, Hanrahan P et al: Are there clinical differences between familial and nonfamilial Alzheimer's disease? Am J Psychiatry 1992; 149:1023-1027

14. Lyketsos CG, Tune LE, Pearlson G, et al: Major depression in Alzheimer's disease. An interaction between gender and family history. Psychosomatics 1996; 37(4):380-384

15. Andreasen NC, Endicott J, Spitzer RL, et al: The family history method using diagnostic criteria: Reliability \& validity. Arch Gen Psychiatry 1997; 34:1229-1235

16. Silverman JM, Raiford K, Edland S et al: The Consortium to Establish a Registry for Alzheimer's disease (CERAD). Part VI. Family history assessment: A multicenter study of first-degree relatives of Alzheimer's disease patients and nondemented spouse controls. Neurology 1994; 44:1253-1259

17. Devi G, Marder K, Schofield PW et al: Validity of family history for the diagnosis of dementia among siblings of patients with late onset Alzheimer's Disease. Genet Epidemiol 1998; 15:215-223

18. Jacobs DM, Sano M, Albert S, et al: Cross-cultural neuropsychological assessment: a comparison of randomly selected, demographically, matched cohorts of English- and Spanish- speaking older adults. J Clin Exp Neuropsychol 1997; 19:331-339

19. McKhann G, Drachman D, Folstein M et al: Clinical diagnosis of Alzheimer's disease: report of the NINCDS-ADRDA Work Group under the auspices of the Department of Health and Human Services Task Force on Alzheimer's Disease. Neurology 1984; 34:939-944

20. Hughes CP, Berg L, Danziger WL, et al: A new clinical scale for the staging of dementia. Br J Psychiatry 1982; 140:566-572

21. Morris JC, McKeel DW, Storandt M, et al: Very mild Alzheimer's disease: informant based clinical, psychometric, and pathologic distinction from normal aging. Neurology 1991; 41:469-478

22. Martin RL, Gerteis J, Gabrielli WF: A family-genetic study of dementia of Alzheimer's type. Arch Gen Psychiatry 1988; 45:894-900

23. Sweet RA, Nimgaonkar VL, Devlin B et al: Increased familial risk of the psychotic phenotype in Alzheimer disease. Neurology 2002; 58:907-911

24. Friedrich U, Mors O, Ewald H: Systematic chromosome examination of two families with schizophrenia and two families with manic depressive illness. Am J Genet. 1996; 67:58-62

25. Byerley W, Hoff M, Holik J et al: Linkage analysis between schizophrenia and index simple-sequence loci for chromosome 21. Hum Hered 1995; 45:49-52

26. Fukuda R, Hattori M, Sasaki $T$ et al: No evidence for a point mutation at codon 713 and 717 of amyloid precursor protein gene in Japanese schizophrenics. Japanese J Hum Gen 1993; 38:407-11

27. Adams RD, Victor M: Huntington chorea. Chapter in Principles of neurology, 5th edition,1993, pp 969-973

28. Pflanz S, Besson JAO, Ebmeier KP, et al: The clinical manifestation of mental disorder in Huntington's Disease: a retrospective case record study of disease progression. Acta Psychiatr Scand 1991: 83: 53-60

29. Tsuang D, DiGiacomo L, Lipe H, et al: Familial aggregation of schizophrenia-like symptoms in Huntington's disease. Am J Med Genet 1998; 81(4):323-327

30. Benton CS, de Silva R, Rutledge SL, et al: Molecular and clinical studies in SCA-7 define a broad clinical spectrum and the infantile phenotype. Neurology 1998; 51(4):1081-1086

31. Farrer LA, O'Sullivan DM, Cupples LA, et al: Assessment of genetic risk for Alzheimer's disease among first-degree relatives. Ann Neurol 1989; 25:485-493

32. Huff JF, Auerbach J, Chakravarti A, et al: Risk of dementia in relatives of patients with Alzheimer's disease. Neurology 1988; 38:786-790

33. Breitner JCS, Silverman JM, Mohs RC, Davis KL. Familial aggregation in Alzheimer's disease: Comparison of risk among relatives of early- and late-onset cases, and among male and female relatives in successive generations. Neurology 1988; 38:207-212

34. Massoud F, Devi G, Stern Y, et al: A clinicopathological comparison of community-based and clinic-based cohorts of patients with dementia. Arch Neurol 1999; 56:1368-1373 